\title{
ESTUDO SOBRE GRAFOS E ALGUMAS APLICAÇÕES
}

Elias de Oliveira Boaventura, Eugenia Brunilda Opazo Uribe

Universidade Federal de Mato Grosso do Sul - UFMS, Curso de Matemática, Três Lagoas - MS. E-mail: oliveiraboaventura@gmail.com

Agência de fomento: Programa de Educação Tutorial

\section{RESUMO}

O presente artigo é resultado da atividade de pesquisa de um aluno do Curso de Matemática no âmbito do Programa de Educação Tutorial, que teve Grafos como tema de estudo, um tema que não é abordado nas disciplinas tradicionais do Curso. Assim, o objetivo principal é apresentar resultados do estudo realizado, abordando conceitos, exemplos e aplicações. A metodologia constou de pesquisa bibliográfica, estudo teórico e de resolução de exercícios, bem como apresentação de seminários. Foram estudados tipos específicos de grafos, correlacionando com exemplos práticos no intuito de ratificar a abrangência e eficácia dessa ferramenta. Foi explorada a origem da Teoria de Grafos, a Fórmula de Euler, bem como a solução de alguns problemas através de interpretação geométrica. Concluímos que, a abordagem histórica e prática é importante como elemento motivacional, enriquecimento intelectual e acadêmico dos alunos, motivando-os a vislumbrar as possíveis aplicações dos conhecimentos adquiridos.

Palavras-chave: Grafos, Fórmula de Euler, Aplicações de Grafos, Ensino de Matemática, Programa de Educação Tutorial.

\section{STUDY ON GRAPH THEORY AND SOME APPLICATIONS}

\begin{abstract}
This article is the result of the research activity of a student in Mathematics Graduation in the Tutorial Educational Program, which had Graphs as a topic of study, a topic that is not covered in the traditional disciplines of the course. Thus, the main objective is to present results of the study, covering concepts, examples and applications. The methodology consisted of bibliographical research, theoretical study and exercises, as well as presentation of seminars. Specific types of graphs have been studied by correlating with practical examples in order to ratify the scope and effectiveness of this tool. Was explored the origin of the theory of Graphs, Euler's formula, as well as the solution of some problems through geometric interpretation. We conclude that the historical and practical approach is important as a motivational element, intellectual and academic enrichment students, motivating them to envision the possible applications of the knowledge acquired.
\end{abstract}

Keywords: Graph, Euler's formula, Graph applications, Math Education, Tutorial Educational Program. 


\section{INTRODUÇÃO}

A teoria dos grafos estuda objetos combinatórios, pois os mesmos são bons modelos para muitos problemas em vários ramos da matemática, da informática, da engenharia, da química, da psicologia e da indústria. O artigo de Leonhard Euler, publicado em 1736, sobre o problema das sete pontes de Königsberg é considerado o primeiro resultado da Teoria dos Grafos. Assim como o problema das pontes, muitos dos problemas sobre grafos tornaram-se célebres porque são um interessante desafio intelectual e porque têm importantes aplicações práticas.

O objetivo do presente trabalho é apresentar resultados do estudo realizado, abordando conceitos, exemplos e aplicações.

\section{METODOLOGIA}

O trabalho foi desenvolvido individualmente através de pesquisa bibliográfica e um estudo teórico que incluiu apresentação de seminários, resolução de exercícios através de interpretações geométricas e aplicação da fórmula de Euler.

\section{RESULTADOS}

Iniciamos o trabalho apresentando o problema das pontes de Königsberg. Parte desta cidade localizava-se em duas ilhas do rio Pregel as quais estavam ligadas às margens de uma à outra através de sete pontes conforme ilustra a (Figura 1).
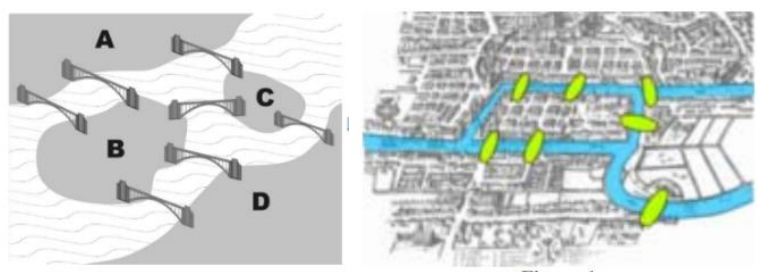

Figura 01. Pontes de Königsberg em 1736.

Fonte: www.google.com.br

O problema teria se originado pela curiosidade dos habitantes de não encontrarem um trajeto (com partida e chegada a um mesmo lugar) que lhes permitisse atravessar apenas uma vez cada uma das pontes. O matemático suiço Leonhard Euler (1707-1783) ao tomar conhecimento deste problema resolveu-o (indicando a impossibilidade da existência de tal percurso, numa memória que publicou em S. Petersburgo em 1736).

Entenderemos por grafo um modelo matemático utilizado para representar relações entre objetos, aplicado na definição ou resolução de problemas de diversas áreas. Os elementos de um grafo são os vértices e as arestas. O vértice, também conhecido como nódulo ou nó, é cada uma das entidades (objetos) representadas em um grafo. A aresta, também chamada de arco, faz a ligação entre cada uma desses vértices, indicando como os mesmos estão relacionados.

Definição: Um grafo $G=(V, E)$ é um conjunto não-vazio $V$, cujos elementos são chamados vértices, e um conjunto $E$ de arestas. Uma aresta é um par não-ordenado $\left(v_{i}, v_{j}\right)$, onde $v_{i}$ e $v_{j}$ são elementos de V.

Por exemplo,

Figura 2. Grafo relação de amizade

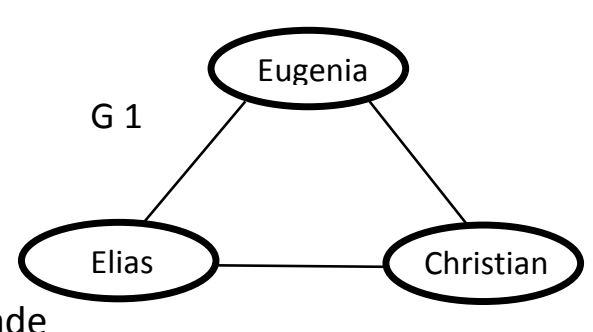

Fonte: Elaborada pelos autores. 
Neste caso, teremos:

$V=\{$ Eugenia, Christian, Elias $\}$

$A=\{$ (Eugenia, Christian), (Eugenia, Elias), (Christian, Elias), (Christian, Eugenia), (Elias, Christian), (Elias, Eugenia) $\}$.

Com relação aos vértices podemos afirmar que, dois vértices $A$ e $B$ são adjacentes quando existe uma aresta ligando-os e esta aresta diz-se ser incidente a ambos. Definiremos também o grau de um vértice como a quantidade de arestas que estão ligadas a este vértice, assim qualquer vértice que tenha grau zero, ou seja, que não tenha nenhuma aresta ligada a ele será chamado vértice isolado.

Com relação às arestas podemos afirmar que, duas arestas são adjacentes quando possuem ao menos um vértice em comum. Duas ou mais arestas são paralelas, quando possuem o mesmo par de vértices. Finalmente, laço é uma aresta que liga um vértice a si mesmo.

Com relação aos Grafos denominaremos: "Passeio entre nós" a sequência alternada entre vértices e arestas, "Caminho ou Caminho Simples" um passeio que não contém nós repetidos. "Comprimento do caminho" ao número de arestas usadas para percorrer o caminho. "Ciclo ou Circuito" a um caminho que começa e termina no mesmo vértice.

TIPOS DE GRAFOS

Tabela 01. Tipos de Grafo, Definição e Representação Geométrica.

\begin{tabular}{|c|c|c|}
\hline Tipos & Definição & Figura \\
\hline Grafo Trivial & $\begin{array}{l}\text { É um grafo com um único } \\
\text { vértice e não tem arestas. }\end{array}$ & 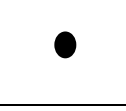 \\
\hline Grafo Simples & $\begin{array}{l}\text { É um grafo não direcionado sem } \\
\text { laços e sem arestas paralelas }\end{array}$ & \\
\hline Grafo Completo & $\begin{array}{l}\text { É um grafo simples onde todos } \\
\text { os vértices conectam-se uns aos } \\
\text { outros }\end{array}$ & \\
\hline Grafo regular. & $\begin{array}{l}\text { É um grafo onde todos os } \\
\text { vértices possuem o mesmo grau }\end{array}$ & \\
\hline Subgrafo & $\begin{array}{l}\text { É um grafo construído de tal } \\
\text { forma que seus vértices e suas } \\
\text { arestas estão contidos em outro } \\
\text { grafo. }\end{array}$ & \\
\hline Grafo Bipartido. & $\begin{array}{l}\text { Um grafo é dito ser bipartido } \\
\text { quando seu conjunto de } \\
\text { vértices V puder ser } \\
\text { particionado em dois } \\
\text { subconjuntos } \mathrm{V} 1 \text { e } \mathrm{V} 2 \text {, tais que } \\
\text { toda aresta de } \mathrm{G} \text { une um vértice } \\
\text { de } \mathrm{V} 1 \text { a outro de } \mathrm{V} 2 \text {. }\end{array}$ & \\
\hline
\end{tabular}




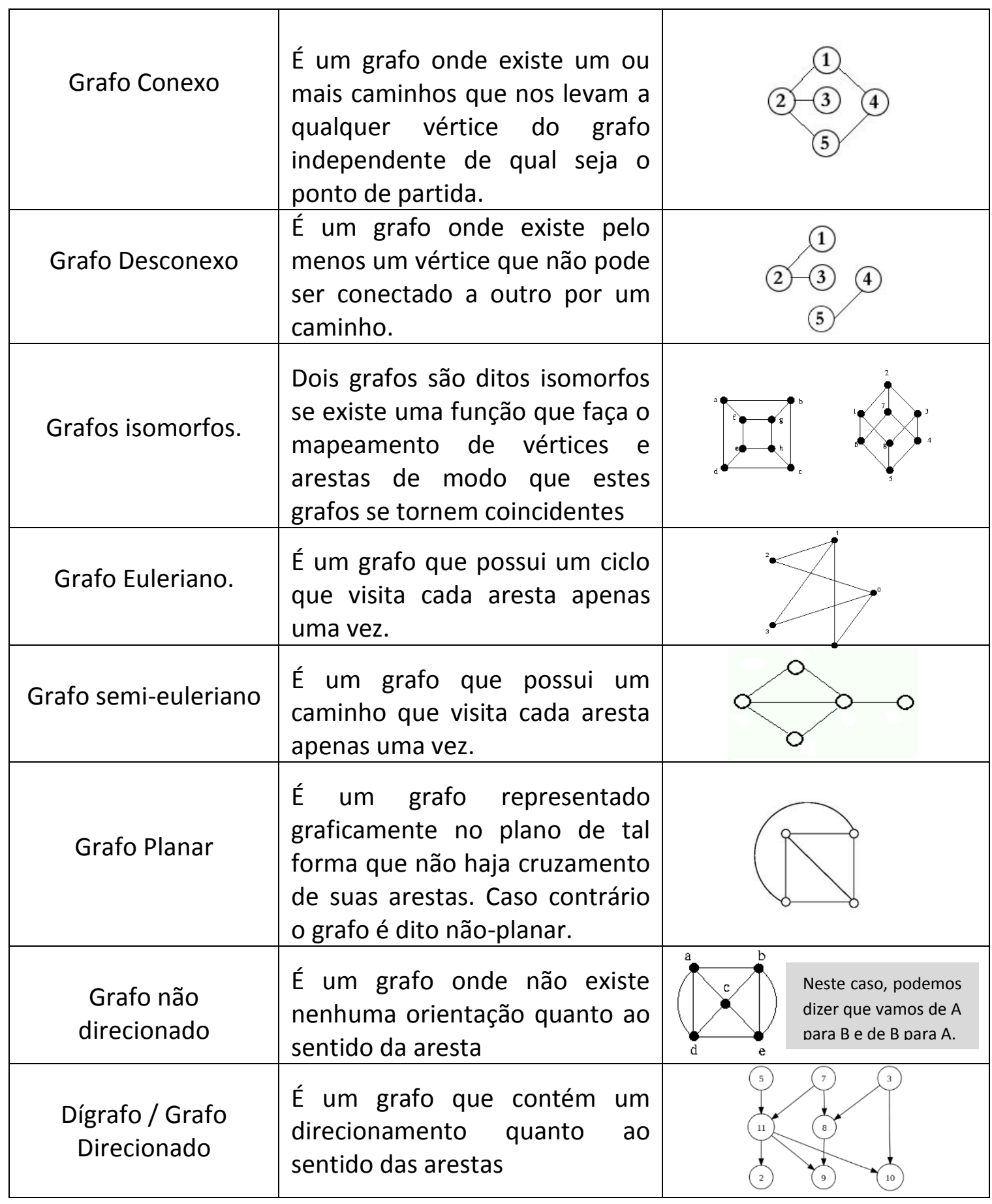

Fonte: Elaborada pelos autores.

Observemos que, em um dígrafo, com relação ao grau de um grafo, podemos determinar:

- Grau de entrada: que é o número de arestas que chegam ao vértice.

- Grau de saída: que é o numero de arestas que partem do vértice.

\section{Exemplo 2:}

\begin{tabular}{|c|}
\hline Grau de Entrada \\
\hline$G(X)=1$ \\
$G(W)=2$ \\
$G(Z)=2$ \\
$G(Y)=1$ \\
\hline
\end{tabular}

\begin{tabular}{|c|}
\hline Grau de saída \\
\hline$G(X)=2$ \\
$G(W)=1$ \\
$G(Z)=1$ \\
$G(Y)=2$ \\
\hline
\end{tabular}




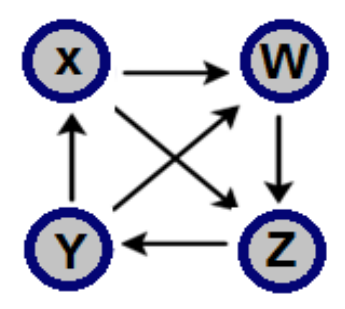

Figura 3. Dígrafo.

Fonte: Elaborado pelos autores.

O Teorema de Euler e seu corolário, que apresentamos a seguir, juntamente com sua fórmula, representam ferramentas importantes para a resolução de problemas associados a grafos.

Teorema de Euler: Um grafo conexo (não necessariamente simples) G é euleriano se e somente se todos os seus vértices tem grau par.

Corolário: Um grafo conexo (não necessariamente simples) G é semi-euleriano se, e somente se, no máximo, dois vértices têm grau ímpar.

Teorema (Fórmula de Euler): Se G é um grafo planar conexo com $V$ vértices, $A$ arestas e $R$ faces, então vale que $\mathrm{N}-\mathrm{M}+\mathrm{F}=2$.

Existem alguns problemas clássicos utilizados para ilustrar e ajudar a entender os conceitos e como fazer as aplicações. Apresentaremos alguns destes exemplos com o objetivo de facilitar o entendimento da aplicação de grafos a problemas mais complexos que serão apresentados posteriormente.

Problema 1. Problema a casinha: Neste exemplo, o desafio consiste em desenhar a casinha, conforme ilustrada na Figura 4, sem tirar o lápis do papel, de modo que não passemos pelo mesmo caminho mais de uma vez, a figura a direita, representa a solução da questão.
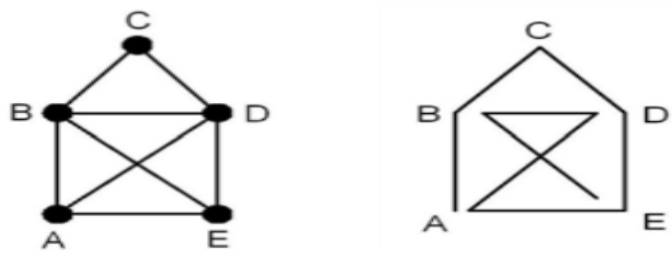

Figura 04. Esboço gráfico do Problema da casinha, juntamente com sua solução.

Fonte: Elaborada pelos autores.

Problema 2. Problema da ligação das 3 casas: O problema propõe ligar Luz, Gás e Telefone a três casas sem que as linhas se cruzem (supondo todas as ligações, fios e canos em um mesmo plano). Solução: Podemos representar geometricamente o problema da seguinte forma:

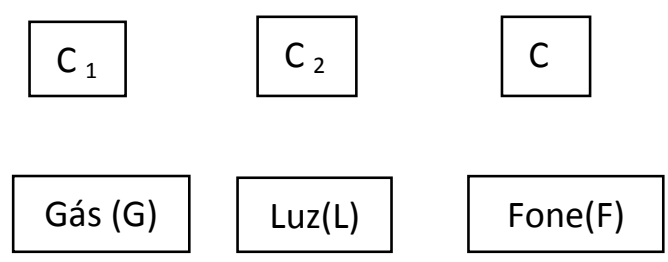

Figura 5. Representação geométrica do problema proposto.

Fonte: Elaborada pelos autores

Considerando $C_{1}, C_{2}, C_{3}$ e $G, L, F$ vértices do grafo que representa o problema e $G_{1}, G_{2}$, $\mathrm{GC}_{3}, \mathrm{LC}_{1}, \mathrm{LC}_{2}, \mathrm{LC}_{3}, \mathrm{FC}_{1}, \mathrm{FC}_{2}, \mathrm{FC}_{3}$ suas respectivas arestas. Verifiquemos através da formula de Euler se este é um grafo planar.

$$
\mathrm{V}-\mathrm{A}+\mathrm{R}=2 \leftrightarrow \mathrm{R}=2-\mathrm{V}+\mathrm{A} \leftrightarrow \mathrm{R}=2-6+9 \leftrightarrow \mathrm{R}=5
$$


Portanto, devemos ter, segundo a fórmula de Euler cinco regiões determinadas pelo grafo, mas como um dessas regiões é ilimitada, temos que ter quatro regiões limitadas pelas arestas deste grafo. Geometricamente temos as seguintes situações:

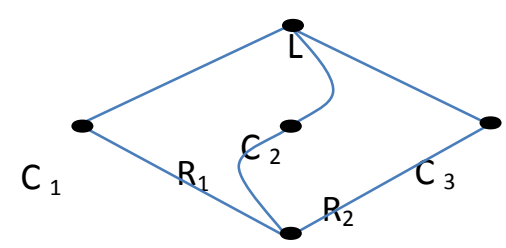

G

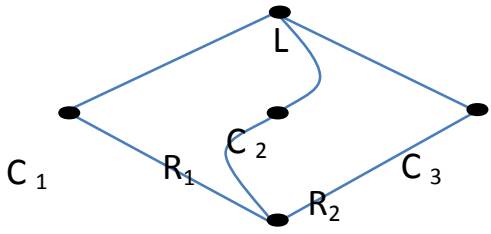

G

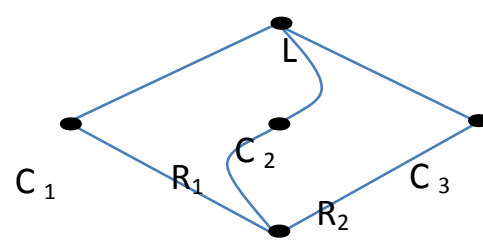

G

Figura 6. Representação geométrica das situações geradas pelo problema proposto. Fonte: Elaborada pelos autores.

Como podemos perceber, por meio da análise gráfica temos que as arestas do grafo determinam 6 regiões, o que vai contra a fórmula de Euler, sendo assim o grafo do problema 2 não é um grafo planar, logo o problema não tem solução.

Problema 3. Problema da coleta de lixo: Suponha que em uma pequena cidade exista apenas um caminhão que realize a coleta de lixo, a fim de otimizar o serviço e reduzir gastos com combustível, o prefeito deseja que o caminhão passe apenas uma vez por cada rua e retorne ao ponto de partida.

Solução: Caso o grafo do mapeamento da cidade fosse tal qual ilustra a figura 7, podemos perceber que qualquer que seja o ponto de partida, temos solução para o problema.

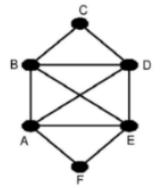

Figura 07. Representação geométrica do problema da coleta de lixo.

Fonte: Elaborada pelos autores

No entanto, caso a cidade possua muito mais ruas e o grafo resultante seja bem mais complexo, como resolver a questão? (Aplicando o teorema de Euler, para analisar a possível solução e implementando uma rotina para calcular qual seria o trajeto a ser percorrido).

Obviamente, podemos utilizar os mesmos conceitos e definições para resolver problemas bem mais complexos, que estão presentes em nosso cotidiano.

Abordaremos duas aplicações importantes para o dia a dia dos habitantes de uma cidade qualquer.

Aplicação I: Quantas linhas de transmissão precisam falhar, no mínimo, para que soframos um apagão? 


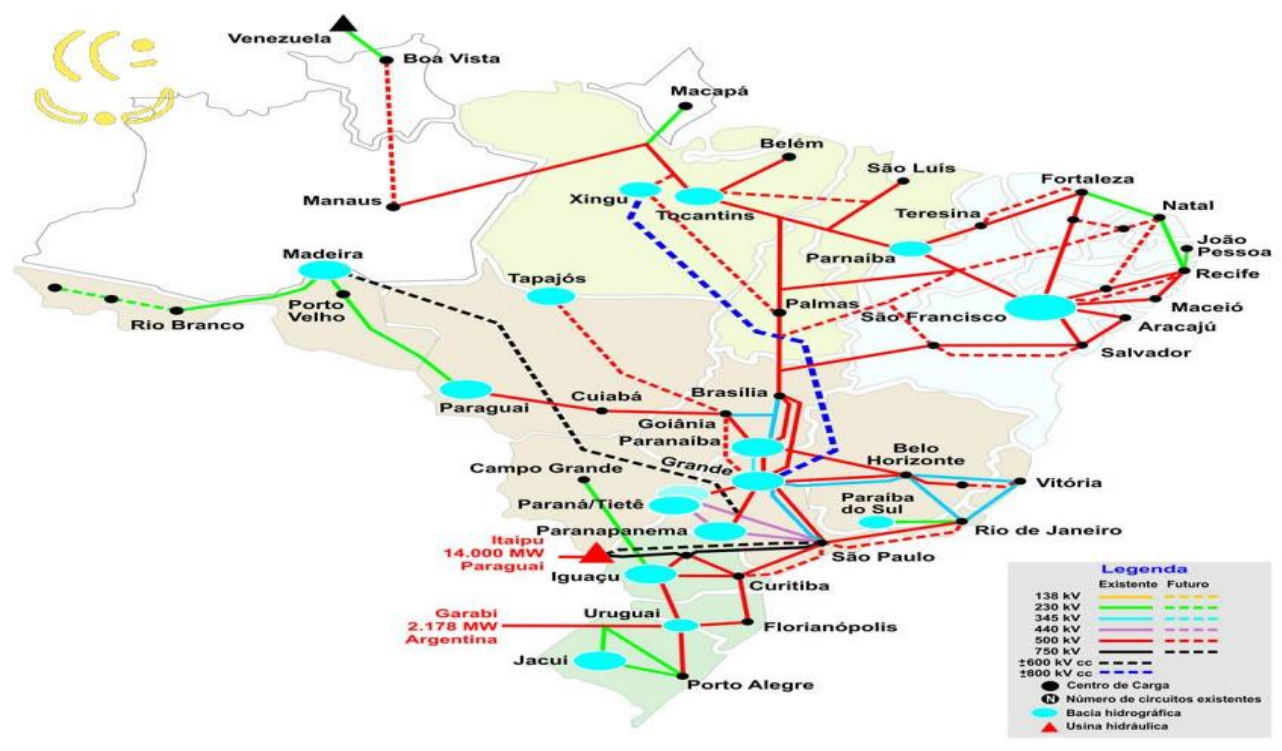

Figura 8. Mapa da rede elétrica do Brasil.

Fonte:https://www.google.com.br/search?q=malha+eletrica+brasileira\&espv=2\&biw=1366\&bih=5 99\&source=Inms\&tbm=isch\&sa=X\&ved=0ahUKEwi9|Y3558bOAhUMkZAKHfMCCdMQ AUIBygC\#im grc=xh-uHnAdrva36M\%3A.

Aplicação II: Rompimento da rede de abastecimento de água da cidade: Nesta aplicação precisa estudar como recompor a rede de abstecimento afetando o mínimo de domicílios possíveis.

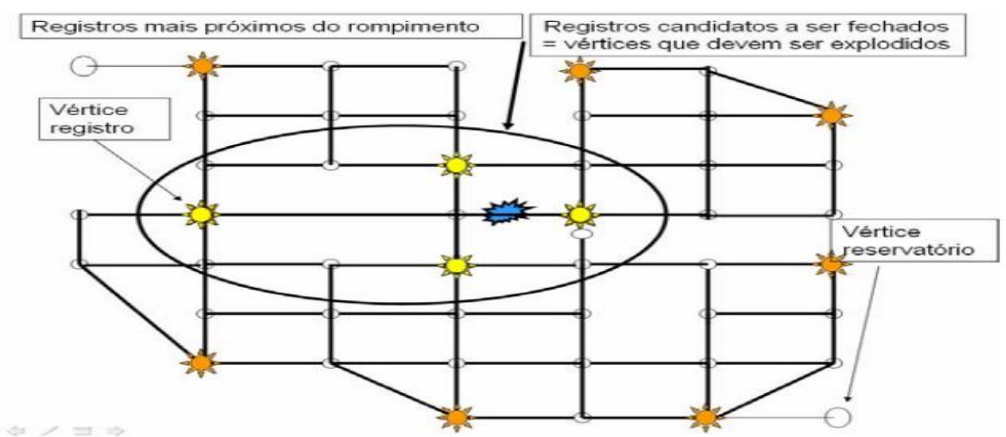

Figura 09. Grafo representativo da rede e água de uma cidade Fonte: Pereira (2008).

\section{DISCUSSÃO}

Através da resolução de problemas de variados níveis de complexidade e seminários apresentados durante o desenvolvimento do trabalho, foi possível verificar a eficiência e praticidade alcançadas por meio da utilização dos conceitos teóricos assimilados durante o estudo de Grafos, bem como na aplicação da fórmula de Euler.

\section{CONCLUSÃO}

Através do presente trabalho foi possível verificar que a Matemática tem um papel fundamental no que diz respeito à solução de problemas dos mais variados tipos ao longo dos tempos, percebemos claramente que a Matemática está entrelaçada com vários outros ramos de estudo, como filosofia, engenharia, química, biologia, computação, entre outras. 


\section{REFERÊNCIAS}

CARDOSO, D. M. Teoria dos Grafos e Aplicações. Departamento de Matemática da Universidade de Aveiro. 2004. Disponível em:

<http://arquivoescolar.org/bitstream/arquivo-e/78/1/TGA2004.pdf>. Acesso: 15/07/2016.

DIESTEL, R. Graph Theory (Graduate Texts in Mathematics 173), Capítulo 4. Springer 1997. Disponível em: <http://www.ime.usp.br/ pf/mac5827/aulas/planar.html>. Acesso: 10/07/2016.

Mariani, A. Teoria dos Grafos. Departamento de Informática e de Estatística. UFSC/CTC/INE. Disponível em: <http://www.inf.ufsc.br/grafos/livro.html>. Acesso: 16/07/2016.

PEREIRA, G. M. R. Algumas aplicações da Teoria de Grafos. FAMAT em Revista. Número 11, outubro de 2008. Disponível em:

<http://www.pucrs.br/famat/viali/graduacao/producao/po_2/literatura/grafos/artigos/Famat_art igo_04.pdf>. Acesso: 13/07/2016.

TAMASSIA, R. Curso de Teoria dos Grafos. Pós Graduação em Ciência da Computação, IME-USP. 2000. Disponível em: <http://www.ime.usp.br/ pf/mac5827/index.html >. Acesso: 10/07/2016. 